\title{
Vitamin D bio-enrichment of pork may offer a food-based strategy to increase vitamin D intakes in the UK population
}

\author{
H. R. Neill ${ }^{1}$, C. I. R. Gill ${ }^{1}$, E. J. McDonald ${ }^{2}$, W. C. McRoberts ${ }^{3}$ and L. K. Pourshahidi ${ }^{1}$ \\ ${ }^{1}$ Nutrition Innovation Centre for Food and Health (NICHE), School of Biomedical Sciences, Ulster University, \\ Coleraine, United Kingdom, BT52 1SA, \\ ${ }^{2}$ Devenish Nutrition Ltd., Lagan House, Belfast, United Kingdom, BT1 $3 B G$ and \\ ${ }^{3}$ Agri-Food and Biosciences Institute, Headquarters, 18 a Newforge Lane, Belfast, BT9 5PX, UK
}

Hypovitaminosis D is prevalent worldwide, with many failing to achieve the recommended nutrient intake (RNI) for vitamin D (10$20 \mu \mathrm{g} / \mathrm{d})^{(1)}$. Moreover, the COVID-19 pandemic has re-emphasised the need to avoid vitamin D deficiency to help maintain immune function ${ }^{(2)}$ and the urgent need for food-based strategies to help address this ${ }^{(3)}$. The aims of the current study were to 1) determine any changes to vitamin D intake and status over a 9-year period, and 2) apply dietary modelling to predict the impact of vitamin D bio-enrichment of pork and pork products on population intakes in the UK.

Data from the National Diet and Nutrition Survey (NDNS) Rolling Programme Year 1-9 (2008/09-2016/17) were analysed using SPSS to determine nationally representative mean vitamin D intakes and $25(\mathrm{OH}) \mathrm{D}$ concentrations [a robust biomarker of vitamin D status]. Subgroup analysis investigating variance in sex, age and season was conducted. Informed by previous studies 4 ), four theoretical dietary modelling scenarios of vitamin D pork bio-enrichment were analysed (vitamin D content $+50 / 100 / 150 / 200 \%$ vs standard).

Vitamin D intake in the UK population has not changed significantly from 2008 to 2017 yet, over the same period significant gender difference (M $2.66 \pm 1.99 \mu \mathrm{g} / \mathrm{d}$ and F $2.30 \pm 1.66 \mu \mathrm{g} / \mathrm{d}, p<0.05)$ and seasonal variation in the mean $25(\mathrm{OH}) \mathrm{D}$ concentrations were evident. In 2016/17, across all age groups, $13.2 \%$ were considered insufficient $(25(\mathrm{OH}) \mathrm{D}$

$<25 \mathrm{nmol} / \mathrm{L}$ ). Across the whole group, theoretical modelling demonstrated an increase of 4.9, 10.1, 15.0 and $19.8 \%$ in daily vitamin D intake when vitamin D concentrations in bio-enriched pork were elevated by 50,100, 150 and $200 \%$, respectively. Based on the $200 \%$ modelling scenario, a greater relative change was observed in males $(22.6 \%)$ compared to females $(17.8 \%)$, and although older adults $(65+$ years) had significantly greater vitamin $\mathrm{D}$ intakes compared to other age categories $(3.28 \pm 2.27 \mu \mathrm{g} / \mathrm{day})$, this age group observed the smallest relative increase from the dietary modelling scenarios $(14.3 \%)$. The greatest relative change was observed amongst 11-18-year olds, where 200\% vitamin D bio- enrichment of pork and pork products would result in a $25.2 \%$ increase in mean daily vitamin $\mathrm{D}$ intakes.

Vitamin D intakes have remained stable in the UK across almost a decade, and current results confirm that strategies are required to help the population achieve the RNI for vitamin D. These findings align with Irish data and provides incentive to pursue bio-enrichment practices. Specifically, bio-enrichment of pork meat provides a proof of concept, demonstrating that animal-based strategies may offer an important contribution to help to improve the vitamin D intakes of the UK population, particularly adolescents.

\section{Acknowledgments}

The authors acknowledge the funding received to conduct this review as part of a $\mathrm{PhD}$ studentship awarded to H.R.N.

\section{References}

1. Spiro A \& Buttriss JL (2014) Nutr Bull 39, 322-350. Lanham-New SA, Webb AR, Cashman KD et al. (2020) BMJ Nutrition, Prevention \& Health [Epub].

2. Cashman KD (2020) Photochem Photobiol Sci [Epub].

3. Larson-Meyer DE, Ingold BC, Fensterseifer SR et al.. (2017) PLoS One 12, e0187877. 